\title{
CCD-based observations of PG $0856+121$ and a theoretical analysis of its oscillation modes ${ }^{\star}$
}

\author{
A. Ulla ${ }^{1}$, M. R. Zapatero Osorio ${ }^{2,3}$, F. Pérez Hernández ${ }^{2,4}$, and J. MacDonald ${ }^{5}$ \\ 1 Universidade de Vigo, Departamento de Física Aplicada, Área de Física da Terra, Astronomía e Astrofísica, \\ Facultade de Ciencias, Campus Lagoas-Marcosende, 36200 Vigo, Spain \\ 2 Instituto de Astrofísica de Canarias, c/ Vía Láctea s/n, 38200 La Laguna, Tenerife, Spain \\ 3 CALTECH, MS 150-21, Pasadena, CA 91125, USA \\ ${ }^{4}$ Departamento de Astrofísica, Universidad de La Laguna, Tenerife, Spain \\ 5 Department of Physics and Astronomy, University of Delaware, Newark, DE 19716, USA
}

Received 5 July 2000 / Accepted 29 January 2001

\begin{abstract}
B V R I$ CCD-based and near-IR $(J)$ imaging, together with unfiltered photometry of the hot subdwarf B star PG 0856+121, are reported. Two close, faint, red, point-like sources are resolved. They account for the previously reported IR excess observed in this hot subdwarf. In addition, the new unfiltered differential photometry of PG 0856+121 confirms its previously reported pulsational nature. A comparison with the oscillation modes of stellar models suggests the presence of g modes.
\end{abstract}

Key words. binaries: close - stars: individual: PG 0856+121 - stars: oscillations - subdwarfs

\section{Introduction}

Hot B-type subdwarfs (sdBs) are H-rich blue subluminous objects with temperatures not exceeding about $35000 \mathrm{~K}$ (Greenstein \& Sargent 1974; Heber 1986). They have a canonical mass of $0.55 M_{\odot}$, with thin H-rich envelopes of less than $0.02 M_{\odot}$, and a distribution in $\log g$ around 5.25-6.5 (Ulla \& Thejll 1998, hereafter referred to as UT98). These objects are proposed to be progenitors of white dwarfs and descendants of blue horizontal branch stars or asymptotic giant branch (AGB) stars (Saffer et al. 1998). They are also proposed to be responsible for the UV upturn flux observed in early-type galaxies (Bica et al. 1996). Among the various theories for the origin and final fate of the sdBs, close binary evolution has been suggested as one of the likely channels. These investigations are relevant to the formation of type Ia supernovae by merging of double-degenerate pairs, in which one or both members could be descendants of hot subdwarfs (Saffer et al. 1998). Enough evidence has been accumulated to date in favor of a binary nature for at least $40 \%$ of the field hot B subdwarf stars (e.g. Allard et al. 1994; Jeffery \& Pollacco 1998;

Send offprint requests to: A. Ulla, e-mail: ulla@uvigo.es

* Based on observations made with the IAC80 Telescope operated on the island of Tenerife by the Instituto de Astrofísica de Canarias in the Spanish Observatorio del Teide.
UT98), with the detected companions ranging broadly in spectral type and physical parameters.

It is therefore very important to continue to seek information on the current binary nature of hot subdwarfs. In this regard and based on $J H K$ photometry, Thejll et al. (1995, hereafter referred to as TUM95) and UT98 have compiled a list of suitable candidates. They also suggested some particular targets for further investigation, despite large error bars associated with the observations (see UT98 for details). A way to pursue a more detailed study of the binary nature of such objects is to obtain filtered CCD imaging to search for close red components whose IR emission could have contributed to TUM95 and UT98 measurements. With that aim we have started such a program and present here results for the sdB star PG $0856+121$. Table 1 summarizes relevant information about this object published to date.

PG 0856+121 was suspected to be a possible pulsating sdB candidate by Koen et al. $(1997,1998 \mathrm{a})$, based on the similarity of its physical properties to those of known sdB pulsating - or EC14026 - stars (Kilkenny et al. 1997). This suspicion was confirmed by Piccioni et al. (2000), who found periodic light variations with frequencies of $2.3 \mathrm{mHz}$ and $3.2 \mathrm{mHz}$ at a reasonable confidence level. We present our CCD and near-IR observations in Sect. 2. Section 3 provides a brief description of the models used 
Table 1. Summary of properties of PG $0856+121$ (references provided)

\begin{tabular}{|c|c|c|c|c|}
\hline Names: & PG $0856+121$ & (1) & WD $0856+121$ & $(2)$ \\
\hline Sp.type: & $\mathrm{sdB}$ & $(1 ; 3)$ & non variable & (4) \\
\hline $\mathbf{R A}(1950)=$ & 085618.8 & Dec $=$ & +120806 & (1) \\
\hline$(2000)$ & 085902.723 & & +115624.73 & (5) \\
\hline $\mathbf{L I I}=$ & 216.56 & $\mathbf{B I I}=$ & 33.67 & (3) \\
\hline$l=$ & 216.49 & $b=$ & 33.68 & (5) \\
\hline distance: & (pc) & 990 & \pm 370 & $(5,6)$ \\
\hline heigh: & (pc) & 550 & \pm 210 & $(3)$ \\
\hline rad. vel.: & $\left(\mathrm{km} \mathrm{s}^{-1}\right)$ & $\begin{array}{l}+85 \\
+97\end{array}$ & \pm 10.2 & $\begin{array}{c}(5,6) \\
(4)\end{array}$ \\
\hline prp. mot.: & $\begin{array}{c}\mu_{\alpha} \cos \alpha \\
-19.4\end{array}$ & $\begin{array}{c}\mu_{\delta} \\
-19.8\end{array}$ & (mas/yr) & $(5,6)$ \\
\hline \multicolumn{5}{|c|}{ orbital components, velocity and parameters: (5) } \\
\hline$X=-9.16$ & $Y=-0.49$ & $Z=0.55$ & $(\mathrm{kpc})$ & \\
\hline$U=-74$ & $V=116$ & $W=-46$ & $\left(\mathrm{~km} \mathrm{~s}^{-1}\right)$ & \\
\hline $\begin{array}{l}\text { It }\left(\mathrm{kpc} \mathrm{km} \mathrm{s}^{-1}\right)= \\
\text { magnitudes: }\end{array}$ & -1099 & ecc $=0.48$ & nze $=0.14$ & \\
\hline$B$ & $V$ & $R$ & $I$ & \\
\hline 13.248 & 13.559 & 13.667 & 13.805 & \\
\hline \pm 0.022 & \pm 0.020 & \pm 0.020 & \pm 0.018 & $(7)$ \\
\hline$B$ & $P_{\text {mag }}$ & $U-B$ & $B-V$ & \\
\hline 13.28 & 13.03 & -1.03 & -0.19 & $(1)$ \\
\hline$v$ & $u-v$ & $g-v$ & $g-r$ & \\
\hline 13.52 & -0.11 & -0.15 & -0.52 & (1) \\
\hline$y$ & $b-y$ & $m 1$ & $c 1$ & \\
\hline 13.47 & -0.094 & +0.106 & -0.004 & $(8)$ \\
\hline 13.50 & -0.116 & +0.113 & +0.021 & $(8)$ \\
\hline 13.473 & -0.095 & -0.094 & +0.035 & \\
\hline \pm 0.012 & \pm 0.004 & \pm 0.005 & \pm 0.007 & (9) \\
\hline 13.495 & -0.116 & +0.113 & $u-b$ & \\
\hline \pm 0.030 & \pm 0.032 & \pm 0.036 & $.015 \pm .021$ & $(10)$ \\
\hline$J$ & $H$ & $K$ & $E(B-V)$ & \\
\hline 13.42 & 13.59 & 13.84 & $\leq 0.025$ & \\
\hline \pm 0.44 & \pm 0.22 & \pm 0.55 & & (11) \\
\hline$f_{6700} / f_{6050}=$ & 0.651 & $f_{7050} / f_{6700}=$ & 0.840 & $(12)$ \\
\hline \multirow[t]{2}{*}{$T_{\text {eff }}:(\mathrm{K})$} & 22000 & 23800 & 33000 & (3) \\
\hline & 26400 & & & (13) \\
\hline $\log (g)$ & 5.1 & (9) & $5.73(Y=0.001)$ & (13) \\
\hline \multicolumn{5}{|c|}{ optical sp.: (9); finder chart: (1) } \\
\hline \multicolumn{5}{|c|}{$\begin{array}{l}\text { 1: Green et al. (1986); 2: McCook \& Sion (1987); 3: Moehler et al. (1990a); } \\
\text { 4: Saffer et al. (1998); 5: de Boer at al. (1997); } 6 \text { : Colin et al. (1994); } \\
\text { 7: this work; 8: Kilkenny et al. (1988); 9: Moehler et al. (1990b); } \\
\text { 10: Wesemael et al. (1992); 11: UT98; 12: Jeffery \& Pollacco (1998); } \\
\text { 13: Saffer et al. (1994). }\end{array}$} \\
\hline
\end{tabular}

and analysis performed to investigate the oscillatory nature of PG0856+121. Our conclusions are presented in Sect. 4 .

\section{Observations and related results}

CCD-based images $(1024 \times 1024$ pixels $)$ in the Johnson $B V R I$ filters were obtained for PG $0856+121$ using the Thomson camera mounted on the Cassegrain focus of the 0.8-m IAC80 telescope (Teide Observatory) on April 13, 1998. The pixel size of the detector is $0.4325^{\prime \prime}$. The night was photometric with an average seeing value around $2^{\prime \prime}$. Raw frames were processed using standard techniques within the $\operatorname{IRAF}^{1}$ (Image Reduction and Analysis Facility) environment, which included bias subtraction, flat-fielding and correction for bad pixels by interpolation with values from the nearest-neighbour pixels. Landolt (1992) standard stars were also observed at various air masses in order to convert instrumental magnitudes into absolute data. Average rms values for the photometric calibration of each filter are as follows: $0.022 \mathrm{mag}$ for $B, 0.020 \mathrm{mag}$ for $V$ and $R$, and $0.018 \mathrm{mag}$ for $I$.

\footnotetext{
${ }^{1}$ IRAF is distributed by National Optical Astronomy Observatories, which is operated by the Association of Universities for Research in Astronomy, Inc., under contract with the National Science Foundation.
} 


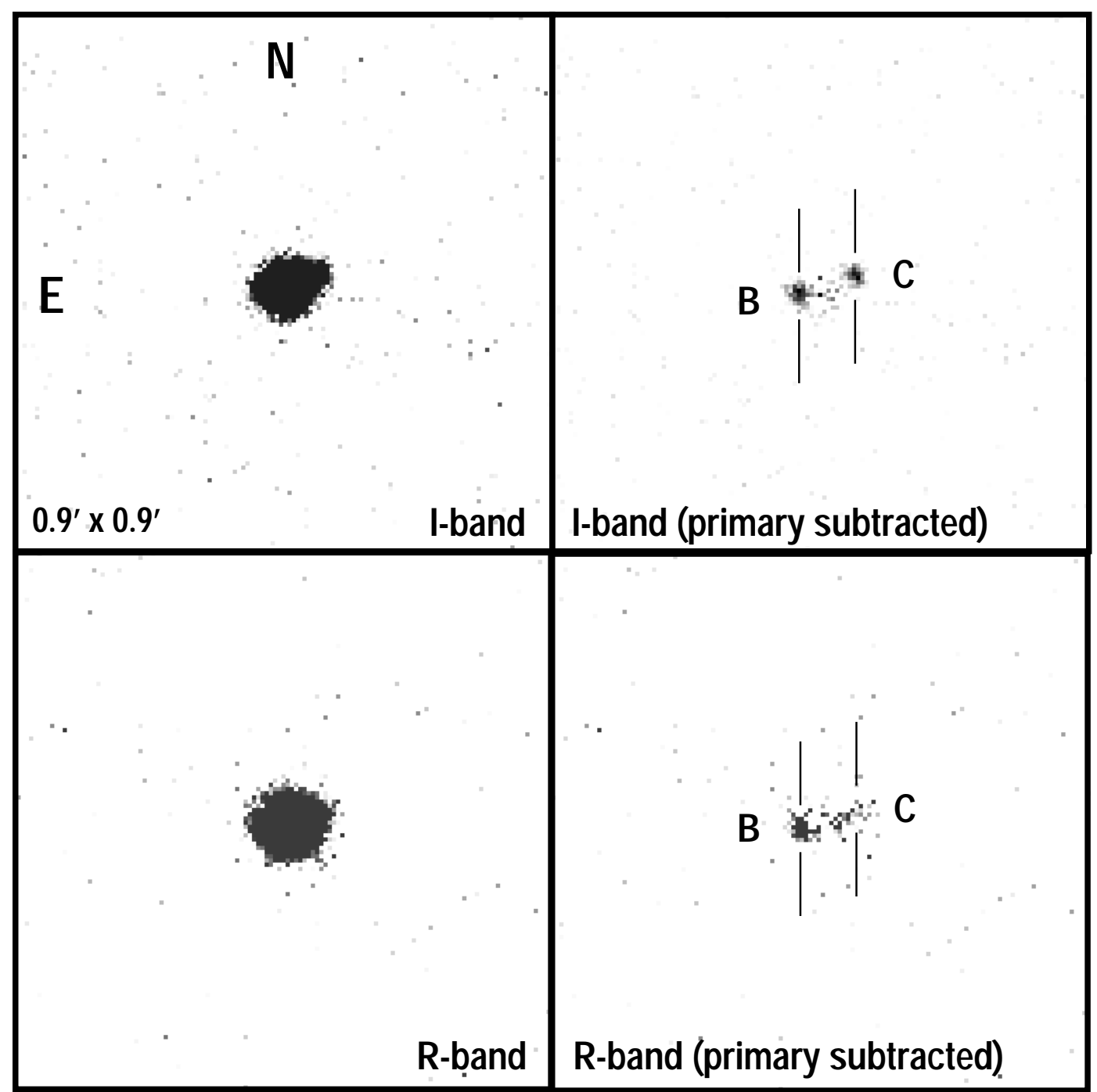

Fig. 1. $R$ - and $I$-band CCD images of PG $0856+121$ in which the locations of the "B" and "C" point-like sources are indicated

Photometry for the target star PG 0856+121 was achieved via the point spread function (PSF) fitting method. The stellar PSF was determined for each colour frame using at least three "isolated", bright point-like sources (not our target) appearing in the IAC80 images, and it was later applied to PG $0856+121$ providing the following colours and magnitudes in the Cousins photometric system: $V=13.559, B-V=-0.311, R=13.667$, $R-I=-0.138$.

Looking at the deconvolved $R$ and $I$ images of PG 0856+121 it became evident that two faint red objects are present very close to the target; one object, named "B" ( $I \leq 17.1, R \leq 17.4)$, located at $\leq 2.4$ " eastward of our star, and another one, named "C" $(I=17.2 \pm 0.05$, $R-I=1.2-1.6)$, located at about $3.5^{\prime \prime}$ northwest $(\mathrm{PA}=$ $296^{\circ}$ ) of it. If object "C" is a Main Sequence star of solar metallicity, a spectral type of M2-M4 would be inferred for it. Figure 1 shows the $R$ and $I$ images of PG $0856+121$ in which the locations of the "B" and " $C$ " point-like sources are indicated. Both sources are quite well detected when subtracting the average PSF from the central target. The typical PSF has FWHM values of $2.2^{\prime \prime}$ and $1.9^{\prime \prime}$ in the $R$ and $I$ frames, respectively. In view of this discovery, we now interpret the suggestion of UT98 for a binary nature of PG $0856+121$ in a different way: as they employed a $15^{\prime \prime}$ aperture for their $J H K$ observations of the target, it is now clear that the two nearby red objects contributed significantly to their measurements. In particular, based on UT98 JHK values for PG 0856+121 (see Table 1) and under the assumption of " $\mathrm{C}$ " being a typical M-type field star, a contribution of $15 \%$ in the $J$ band and of about $40 \%$ in the $K$ band can be estimated. To test whether either of the two nearby objects is gravitationally linked to PG $0856+121$ by taking long-term radial velocity data sets is beyond the scope of the present work. In any case, we might be dealing with a detached system whose long period and orbital parameters should be tested against close binary evolution theories proposed for the hot subdwarfs (see, e.g., Iben \& Tutukov 1986a,b; Iben 1990).

On November 25, 2000, with the goal of proving objects "B" and "C", J images of PG $0856+121$ were obtained with the near-infrared camera $(\mathrm{Hg} \mathrm{Cd}$ Te detector, 


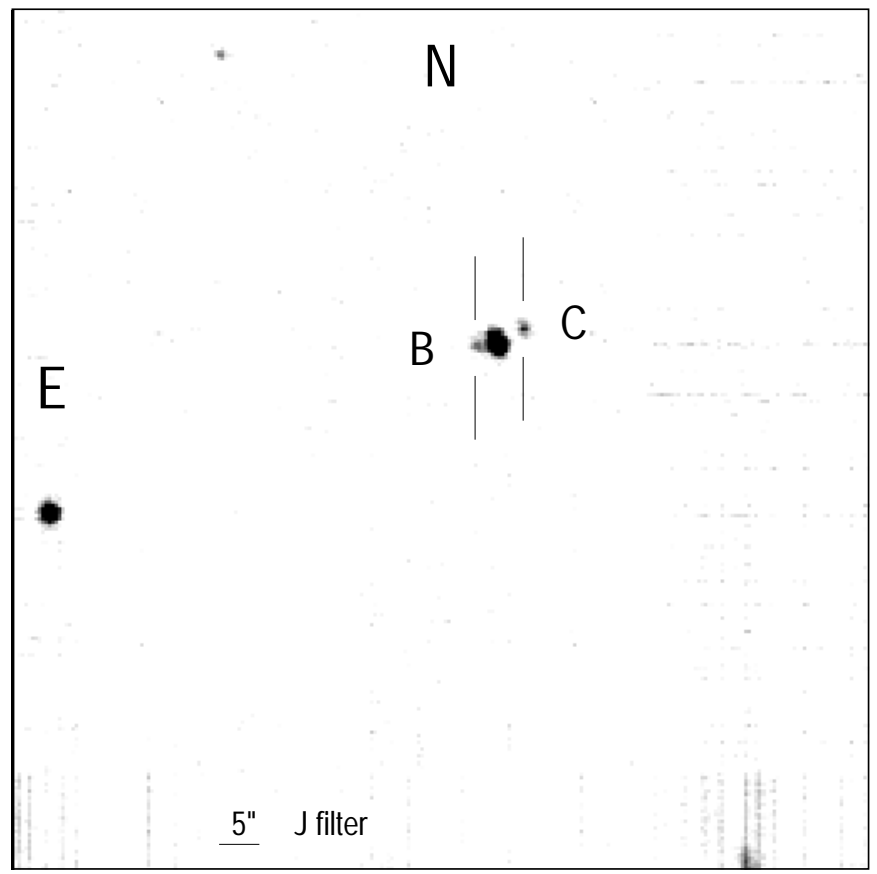

Fig. 2. $J$-band image of PG0856+121 (central, bright star) confirming the presence of two nearby, faint sources labeled as "B" and "C" (see text)

$256 \times 256$ ) mounted on the Cassegrain focus of the 1.5-m Carlos Sánchez Telescope (Teide Observatory). We performed the observations through the "narrow-optics" of the instrument which provides a pixel projection of $0.4^{\prime \prime}$ onto the sky. The atmospheric seeing conditions during the night of the observations were fairly stable around $1.3^{\prime \prime}$. The total integration time was $600 \mathrm{~s}$, the final $J$ image (Fig. 2) being the co-addition of five dithered exposures of 120 s each. Objects "B" and "C" around PG 0856+121 are clearly resolved in this image, and thus proved to be real. The astrometric measurements carried out on this frame confirm those of the IAC80 observations for object "C" and provide for object "B" a separation of $2.3^{\prime \prime}$ at a position angle of $98^{\circ}$ from PG $0856+121$. Instrumental photometry has been performed using a similar procedure as described above and calibrated into real magnitudes with the observations of the standard star AS19-1 (Hunt et al. 1998), which were taken with the same instrumental configuration just after our target. The combined $J$ magnitude of the three sources (PG 0856+121 and objects "B" and " $\mathrm{C}$ ") is $13.89 \pm 0.10 \mathrm{mag}$; this value is in agreement within the error bars of the nearinfrared photometry given in UT98 for PG0856+121. Nevertheless, we have derived the $J$ photometry for each star: $14.16 \mathrm{mag}$ (78.3\% is the contribution to the combined flux) for PG 0856+121, $16.59 \mathrm{mag}$ (8.3\%) for object "B", and $16.08 \mathrm{mag}(13.3 \%)$ for object "C". This latter object is the reddest of the three, and its contribution to the light in the near-infrared is indeed significant. Our $J$ data confirms object "C" as an early- to mid-M type dwarf, and would place it at a distance of $750 \pm 350 \mathrm{pc}$ (adopting a main sequence calibration). This is only marginally

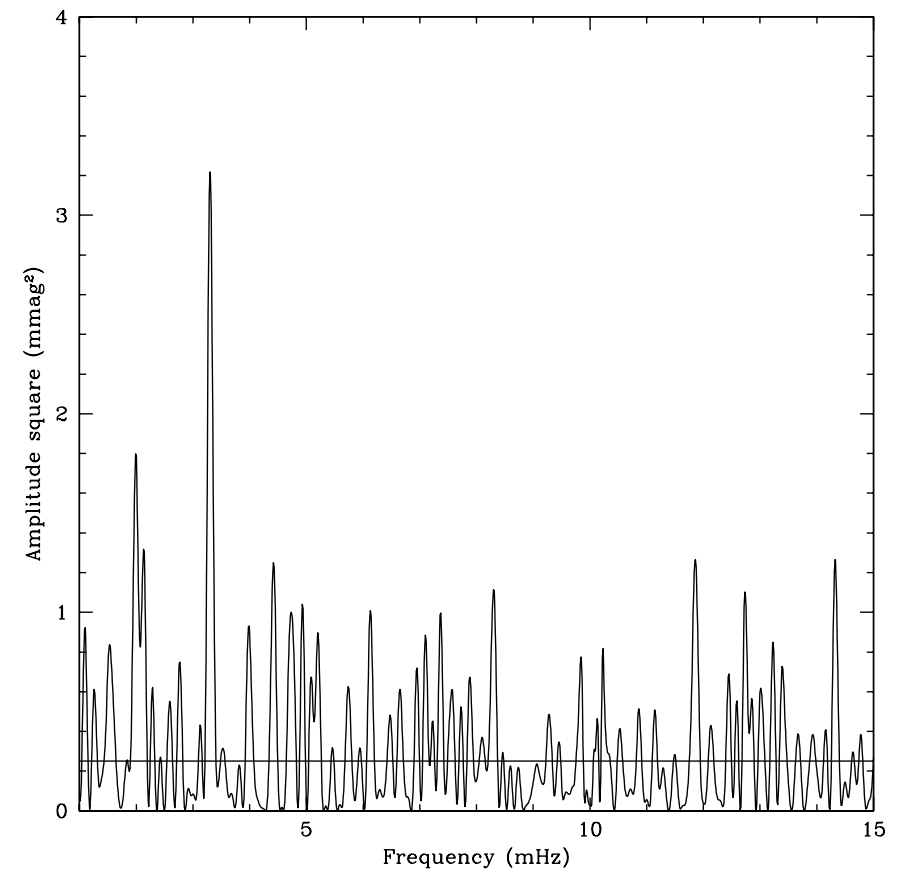

Fig. 3. Frequency spectrum for PG $0856+121$

consistent with the estimated distance of PG $0856+121$. The $I-J$ colour of the hot subdwarf PG0856+121 is now -0.36 mag, which compares well to typical colours of other B type hot subdwarfs.

Unfiltered CCD photometry of PG $0856+121$ was also performed on February 27, 2000, using the same camera and telescope as for our previous observations (on April 13, 1998). The target was monitored every $37 \mathrm{~s} \mathrm{(25} \mathrm{s}$ integration time plus overheads) during 2.4 hours in the airmass interval 1.04-1.20. The CCD detector was windowed so that two comparison stars of similar brightness in the field were observed simultaneously. We performed differential photometry of our target with an accuracy of the order of $0.005 \mathrm{mag}$. In brief, the procedure was as follows: apertures for PG $0856+121$ and the two reference stars were defined as a function of the average FWHM of the frame, and the sky intensity was set as an outer ring of width 1.5 pixel. We compared the reference stars against each other and found them to be constant at the level of our $1 \sigma$ photometric error bars.

Spectral analysis of the February 27, 2000, data was achieved using the ISWF method over the frequency range $1 \mathrm{mHz}$ to $15 \mathrm{mHz}$. The frequency spectrum is shown in Fig. 3 (amplitude square versus cyclic frequency). The horizontal line is the square of the mean value of the amplitude in the frequency range from $1 \mathrm{mHz}$ to $15 \mathrm{mHz}$. The frequency resolution is $0.12 \mathrm{mHz}$. We found a significant peak at $3.30 \mathrm{mHz}(303 \mathrm{~s})$ with a signal-to-noise ratio of 3.6 in amplitude (roughly a 99\% confidence level). This frequency peak does not differ within uncertainties from that derived by Piccioni et al. (2000), i.e. $3.2 \mathrm{mHz}$. This confirms the oscillatory nature of PG 0856+121. Although with a smaller confidence level, peaks at $2.13 \mathrm{mHz}(469.5 \mathrm{~s}$, signal-to-noise of 2.3 in amplitude), and $1.99 \mathrm{mHz}(502.5 \mathrm{~s}$, 
signal-to-noise of 2.7 in amplitude) can be related to the one found by Piccioni et al. (2000) at $2.3 \mathrm{mHz}$ with a similar signal-to-noise level. However, given the confidence level of our own measurements and those of Piccioni et al. (2000), we caution that the reliability of the shorter frequencies should be confirmed by further observations.

\section{Pulsational model calculations}

Oscillations in hot sdB stars have now been well established by observations (see O'Donoghue et al. 1998 and references therein). Potentially, this allows analysis of the internal structure of sdB stars by comparison of the observed frequencies with those corresponding to stellar models with different physical assumptions. Some work has already been done in this direction (see, e.g., Charpinet et al. 1997; Billeres et al. 1998; or Ulla et al. 1999).

Here we compare the frequency peaks reported in the previous section and those found by Piccioni et al. (2000) with theoretical frequencies based on stellar structure models compatible with the surface parameters of PG 0856+121. We have computed stellar structure models of different masses, suitable for the sdB star PG 0856+121. The equation of state, opacity and nuclear reactions are briefly described in Jiménez \& MacDonald (1996). An additional change is the use of OPAL95 opacity tables (Iglesias \& Rogers 1996). These models have helium cores and thin H-rich envelopes. A summary of the models here considered is given in Table 2 . To produce surface abundances similar to those in PG $0856+121$, we have included gravitational settling and element diffusion (Iben \& MacDonald 1985) in models 4, 6 and 8. The envelope compositions for models 1 and 2 are $X=0.71, Y=0.29$, $Z=0.0001$ and for models 3,5 and $7 X=0.60, Y=0.38$, $Z=0.02$. For the models with diffusion the initial envelope composition is also $X=0.60, Y=0.38, Z=0.02$. Gravitational settling causes helium and heavy elements to quickly sink below the photosphere. The outer layers are then pure hydrogen. The fact that $n(\mathrm{He}) / n(\mathrm{H})=0.01$ in PG $0856+121$ and other sdBs can be explained by the presence of a wind that counters the effects of gravitational settling. The wind mass loss rates required to do this are quite small $\left(10^{-15_{-}} 10^{-14} M_{\odot} /\right.$ year $)$ and completely undetectable up to date with ordinary techniques and instrumentation.

The models eigenfrequencies were computed in the adiabatic approximation, using the code developed by Christensen-Dalsgaard (see Christensen-Dalsgaard \& Berthomieu 1991). For models with similar internal structure, as in the present case, the dynamical time scale $t_{\mathrm{dyn}}=\left(R^{3} / G M\right)^{1 / 2}$ dominates the variation of the oscillation frequencies. Thus it is convenient to compare our results in terms of dimensionless frequencies $\sigma$, defined by

$\sigma \equiv\left(\frac{R^{3}}{G M}\right)^{1 / 2} \omega$,

where $\omega$ is the angular oscillation frequency and the other symbols have their conventional meanings.

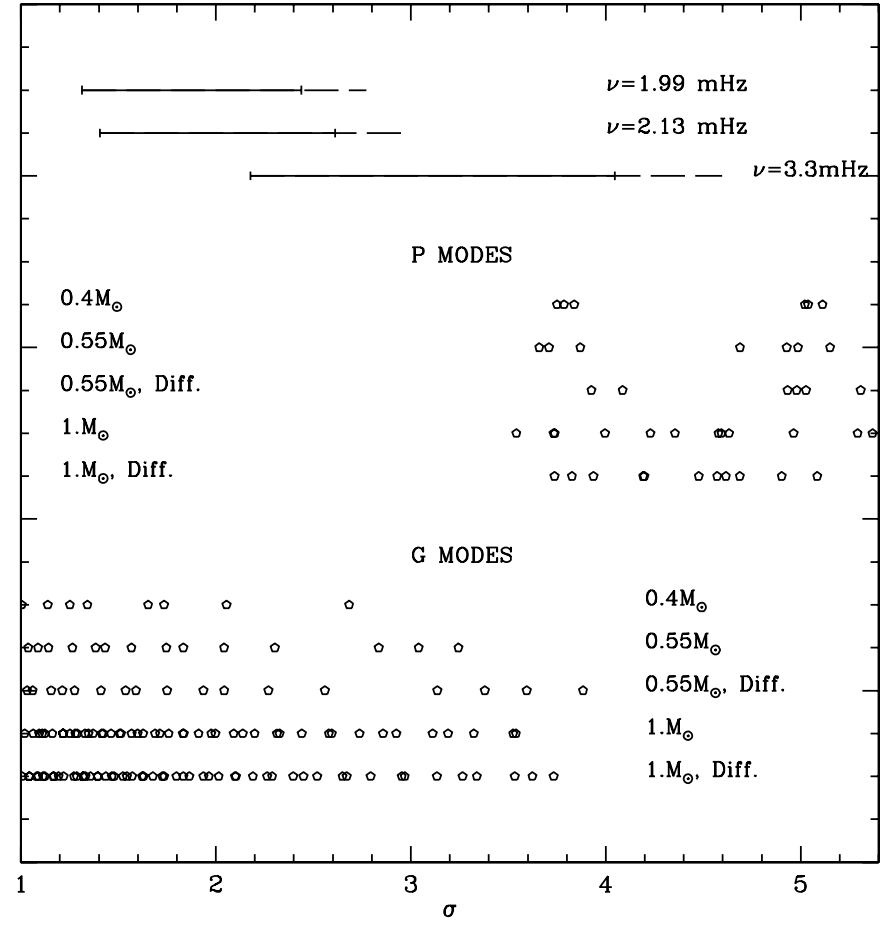

Fig. 4. Dimensionless frequencies for PG $0856+121$. Only 5 models, of those in Table 2, are shown for clarity

In Fig. 4 we show the theoretical dimensionless frequencies, computed by using Eq. (1). Only modes with $\ell \leq 2$ are considered since modes of higher degree can hardly be observed for point-like stars. For the model with $0.4 M_{\odot}$, the 6 p-mode frequencies shown in Fig. 4 correspond to the fundamental and first overtone of the $\ell=0,1,2$ degrees. For other models, the p-mode spectrum is more complex due to the presence of g-like modes. As it can be seen in the figure, the dimensionless frequencies decrease with mass and increase when diffusion is considered.

The dimensionless frequencies corresponding to the observational periods are usually estimated by expressing $\sigma$ in terms of $T_{\text {eff }}, \log g$ and the luminosity $L$ of the stars. However, for this kind of star it seems better to use some estimate of the mass rather than of $L$. In fact, the distance quoted in Table 1 was obtained by assuming the canonical mass for sdB stars. The relations are

$\sigma=\left(\frac{L}{4 \pi \sigma_{\mathrm{SB}}}\right)^{1 / 4} \frac{\omega}{g^{1 / 2} T_{\mathrm{eff}}}=\left(\frac{G M}{g^{3}}\right)^{1 / 4} \omega$

where $\sigma_{\mathrm{SB}}$ is the Stefan-Boltzmann constant.

In particular, for PG 0856+121, we have used the value $\log g=5.73 \pm 0.15$ (Saffer et al. 1994). For the mass we use the canonical value $0.5 \pm 0.1 M_{\odot}$ (Saffer et al. 1994). In addition, the observational frequencies have associated errors, but they are negligible when compared to those of $\log g$ and $M$. The resulting dimensionless frequencies for the observed frequency peaks reported here are shown at the top of Fig. 4 as horizontal continuous lines. Use of the results of Piccioni et al. (2000) instead of those shown 
Table 2. Properties of sdB models 1 through 8, suitable for PG $0856+121$

\begin{tabular}{cccccccc}
\hline $\begin{array}{c}\text { Model } \\
\text { nr. }\end{array}$ & $\begin{array}{c}\text { Mass } \\
M_{\odot}\end{array}$ & $\log g$ & $\begin{array}{c}\text { Envlp. Mass } \\
M_{\odot}\end{array}$ & $\begin{array}{c}\text { Radius } \\
x 10^{9} \mathrm{~cm}\end{array}$ & $\begin{array}{c}\text { Central } \rho \\
x 10^{4} \mathrm{gr} / \mathrm{cm}^{3}\end{array}$ & $\begin{array}{c}T_{\text {eff }} \\
x 10^{4} \mathrm{~K}\end{array}$ & $\begin{array}{c}\text { Luminosity } \\
L_{\odot}\end{array}$ \\
\hline 1 & 0.3999 & 5.843 & $3.1810^{-3}$ & 8.724 & 3.329 & 2.641 & 6.891 \\
2 & 0.4517 & 5.644 & $6.4610^{-3}$ & 11.69 & 2.550 & 2.642 & 12.42 \\
3 & 0.5563 & 5.401 & $6.110^{-3}$ & 17.12 & 1.699 & 2.651 & 26.94 \\
4 & 0.5563 & 5.340 & $5.010^{-3}$ & 18.37 & 1.699 & 2.560 & 26.97 \\
5 & 0.7941 & 4.862 & $2.4310^{-2}$ & 38.05 & 0.941 & 2.696 & 142.4 \\
6 & 0.7941 & 4.821 & $2.0710^{-2}$ & 39.88 & 0.941 & 2.644 & 144.7 \\
7 & 1.0608 & 4.390 & $5.0610^{-2}$ & 75.75 & 0.611 & 2.629 & 510.6 \\
8 & 1.0608 & 4.312 & $4.5310^{-2}$ & 82.83 & 0.612 & 2.541 & 532.7 \\
\hline
\end{tabular}

in the figure, does not change the following discussion. The horizontal dashed lines in Fig. 4 were obtained by assuming the same uncertainty in $\log g$ as quoted above but for a mass range of $0.4 M_{\odot}<M<1 M_{\odot}$. This allows us to explore the possibility that PG $0856+121$ has a mass substantially larger than the canonical one.

In the previous analysis the frequency splittings caused by rotation have been neglected. Although we do not know the rotational velocity of this particular star, we shall consider the value $90 \mathrm{~km} \mathrm{~s}^{-1}$ as an upper limit. This follows from the work by Saffer et al. (1994; and a private communication), who measured this quantity for about $50 \mathrm{sdB}$ stars and none was found to be rotating faster than about $90 \mathrm{kms}^{-1}$. Then, by using the stellar radii given in Table 2, it can be seen that the value of the rotational frequency is, at most, $5 \%$ that of the observational frequencies and, hence, first order corrections for the frequency splitting will be enough for our purposes. By using the values of $M$ and $R$ in Table 2, a rotational frequency splitting $\beta_{\mathrm{nl}} m \sigma_{\text {rot }}$ (here $m$ is the azimuthal order, $\sigma_{\text {rot }}$ the dimensionless rotational angular frequency and the parameter $0 \leq \beta_{\mathrm{nl}} \leq 1$ ) smaller than 0.25 is found for modes with $\ell \leq 2$. Considering this additional uncertainty in Fig. 4 also does not change the conclusions given below.

From Fig. 4 it follows that the peak at $3.3 \mathrm{mHz}$ can be either a g mode or a p mode. Since in other sdB stars only p modes are detected (see e.g. Billeres et al. 1998; Koen et al. 1998b) in agreement with the theoretical expectations of Fontaine et al. (1998) for the EC14026 stars, the latter possibility can be considered with preference. In this case, and assuming the canonical mass, the peak at $3.3 \mathrm{mHz}$ would be a fundamental $\mathrm{p}$-mode with degree $\ell=0,1$, or 2 . On the other hand, if any of the peaks at 1.9 and $2.1 \mathrm{mHz}$ are real and the photometric value of $\log g$ is correctly determined, from Fig. 4 it follows that these peaks must be g-modes of low order. It is important to note that this conclusion does not depend on the details of the model structure, but only on the stellar parameters. The basic reason is that the dimensionless frequencies $\sigma$ of the p-modes are, in a first approximation, independent of such details.

\section{Summary and conclusions}

Optical $(B V R I)$ and near-IR $(J)$ imaging of the field nearby the sdB star PG $0856+121$ revealed the presence of two faint red objects very close $\left(\leq 4^{\prime \prime}\right)$ to the target. In view of this discovery, contamination by them in the nearIR bands is here proposed as the most likely interpretation for the previously reported $J H K$ values for the object (UT98). Our photometric data show that the optical and near-IR colours of PG $0856+121$ are consistent with those of other single hot subdwarfs. Whether either of the two red objects is gravitationally linked to PG $0856+121$ has not been investigated further but it is now brought to the attention of potentially interested researchers. If a binary nature could be established for the target, a refinement in the determination of its physical properties together with those of its companion would be obtainable, in the ways abundantly documented in the literature already. It is worth noting that if the given distance (Table 1) of 990 pc to the target is correct, then the reddest "C" companion, for which an early- to mid-M type dwarf has been determined given its IR colours, would be placed at a distance of $750 \pm 350$, marginally consistent with the estimated distance of PG $0856+121$ above. This makes it very difficult to measure radial velocities by the usual spectroscopic techniques. On the other hand, the presence of an even closer (and therefore unresolved) companion to PG $0856+121$ could still be revealed through radial velocity measurements. As a further suggestion, checking of the eventual binary nature of PG $0856+121$ towards either the "C" or "B" objects, could also be possible on an approximate time-scale of 10 years by means of its proper motion values, as provided by de Boer et al. (1997) and Colin et al. (1994).

Recently, PG $0856+121$ has been reported to display a pulsating nature by Piccioni et al. (2000). The new differential photometry of the target presented here mostly confirms the peaks, at 2.3 and $3.2 \mathrm{mHz}$, detected by these authors. In both works, the largest frequency peak is found at $3.3 \pm 0.1 \mathrm{mHz}$ and, also in both works, peaks around 2.0-2.3 $\mathrm{mHz}$ are found, although with a lower confidence level. We have compared these frequency peaks with those 
of stellar models compatible with the physical properties of the target star. Our results indicate that the peak at $3.3 \mathrm{mHz}$ is a $\mathrm{p}$ - or g-mode with a low radial order; in particular, if the p-mode character is assumed and the canonical mass is considered, it would be the fundamental mode with degree $\ell=0,1$ or 2 .

However, the other frequency peaks are in the g-mode range of our models. Since other pulsating sdB stars seem to have only p-modes, in agreement with earlier theoretical computations, alternative explanations for our low frequency peaks need to be considered. First, the $\mathrm{S} / \mathrm{N}$ ratio for these peaks is rather small and, hence, require further confirmation. Also we note that the results are based on the stellar parameters provided by Saffer et al. (1994) - mainly the value of $\log g-$. To search for systematic shifts in these parameters would demand not only spectra of higher $\mathrm{S} / \mathrm{N}$ ratio but also to test effects such as the importance of considering NLTE atmospheric models for the sdB stars. In view of the importance of these considerations for the particular case of PG $0856+121$, we propose it as a candidate for future improved spectroscopic studies in an attempt to further constrain its oscillatory properties. On the other hand, we find it unlikely that this result arises from errors in the stellar models analysis performed; in particular, as indicated in Sect. 3, even if the models considered were unsuitable for the target, the p-mode range of frequencies has a small dependence on the models' details, and a broad mass range (up to $1 M_{\odot}$ ) has been tested still yielding low frequency peaks in the g-mode range.

Acknowledgements. The authors are grateful to V. J. S. Béjar for his assistance in obtaining the $J$ images at the CST on November 25, 2000. Ana Ulla acknowledges support from the Spanish MEC DGESIC under contract PB97-1435-C02-02 and from the Spanish MCT DGI under contract AYA2000-1691. This research made use of the SIMBAD database, operated at CDS, Strasbourg, France and of the NASA Astrophysics Data System (ADS).

\section{References}

Allard, F., Wesemael, F., Fontaine, G., Bergeron, P., \& Lamontagne, R. 1994, AJ, 107, 1565

Bica, E., Bonatto, C., Pastoriza, M. G., \& Alloin, D. 1996, A\&A, 313, 405

Billeres, M., Fontaine, G., Brassard, P., et al. 1998, ApJ, 494, L75

Charpinet, S., Fontaine, G., Brassard, P., \& Dorman, B. 1997, ApJ, 489, L149
Christensen-Dalsgaard, J., \& Berthomieu, G. 1991, in Solar Interior and Atmosphere, Space Science Series, ed. A. N. Cox, W. C. Livingston, \& M. Mathews (Univ. Arizona Press), 401

Colin, J., de Boer, K. S., Dauphole, B., et al. 1994, A\&A, 287, 38

de Boer, K. S., Aguilar Sánchez, Y., Altmann, M., et al. 1997, A\&A, 327, 577

Fontaine, G., Charpinet, S., Brassard, P., et al. 1998, in IAU Sym. 185, New Eyes to See Inside the Sun and Stars, ed. F.-L. Deubner, J. Christensen-Dalsgaard, \& D. Kurtz, 367 Green, R. F., Schmidt, M., \& Liebert, J. 1986, ApJS, 61, 305 Greenstein, J. L., \& Sargent, A. I. 1974, ApJS, 28, 157

Heber, U. 1986, A\&A, 155, 33

Hunt, L. K., Mannucci, F., Testi, L., et al. 1998, AJ, 115, 2594

Iben, I. Jr. 1990, ApJ, 353, 215

Iben, I. Jr., \& MacDonald, J. 1985, ApJ, 296, 540

Iben, I. Jr., \& Tutukov, A. V. 1986a, ApJ, 311742

Iben, I. Jr., \& Tutukov, A. V. 1986b, ApJ, 311753

Iglesias, C. A., \& Rogers, F. J. 1996, ApJ, 464, 943

Jeffery, C. S., \& Pollacco, D. L. 1998, MNRAS, 298, 179

Jiménez, R., \& MacDonald, J. 1996, MNRAS, 283, 721

Kilkenny, D., Heber, U., \& Drilling, J. 1988, SAAO Circ. No. 12, and later electronic versions thereof distributed by the authors

Kilkenny, D., Koen, C., O'Donoghue, D., \& Stobie, R. S. 1997, MNRAS, 285, 640

Koen, C., Kilkenny, D., O’Donoghue, D., \& Stobie, R. S. 1998a, in IAU Sym. 185, New Eyes to See Inside the Sun and Stars, ed. F.-L. Deubner, J. Christensen-Dalsgaard, \& D. Kurtz, 361

Koen, C., Kilkenny, D., O'Donoghue, D., Van Wyk, F., \& Stobie, R. S., 1997, MNRAS, 285, 645

Koen, C., O'Donoghue, D., Pollacco, D. L., \& Nitta, A. 1998b, MNRAS, 300, 1105

Landolt, A. U. 1992, AJ, 104, 340

McCook, G. P., \& Sion, E. M. 1987, ApJS, 65, 603

Moehler, S., Heber, U., \& de Boer, K. S. 1990a, A\&A, 239, 265

Moehler, S., Richtler, T., de Boer, K. S., Dettmar, R. J., \& Heber, U. 1990b, A\&AS, 86, 53

O'Donoghue, D., Koen, C., Solheim, J.-E., et al. 1998, MNRAS, 296, 296

Piccioni, et al. 2000, A\&AL, 354, 13

Saffer, R., Bergeron, P., Koester, D., \& Liebert, J. 1994, ApJ, 432, 351

Saffer, R. A., Livio, M., \& Yungelson, L. R. 1998, ApJ, 502, 394

Thejll, P., Ulla, A., \& MacDonald, J. 1995, A\&A, 303, 773

Ulla, A., \& Thejll, P. 1998, A\&AS, 132, 1

Ulla, A, Thejll, P., Pérez Hernández, F., MacDonald, J., \& Lawlor, T. 1999, in 11th European Workshop on White Dwarfs, ASP Conf. Ser. 169, ed. J.-E. Solheim, \& E. G. Meistas, 58

Wesemael, F., Fontaine, G., Bergeron, P., Lamontagne, R., \& Green, R. F. 1992, AJ, 104, 203 